\title{
BMJ Open Quality Improving the management of type 2 diabetes through large-scale general practice: the role of a data-driven and technology-enabled education programme
}

Tarek F Radwan, ${ }^{1}$ Yvette Agyako, ${ }^{1}$ Alireza Ettefaghian, ${ }^{1}$ Tahira Kamran, ${ }^{1}$ Omar Din, ${ }^{1}$
Mohammad Aumran Tahir, ${ }^{1}$ Peter Schofield, ${ }^{2}$ Veline L'Esperance ${ }^{1,2}$

To cite: Radwan TF, Agyako Y, Ettefaghian $A$, et al. Improving the management of type 2 diabetes through largescale general practice: the role of a data-driven and technology-enabled education programme. BMJ Open Quality 2021;10:e001087. doi:10.1136/ bmjoq-2020-001087

Received 30 June 2020 Revised 28 November 2020 Accepted 13 January 2021
Check for updates

(C) Author(s) (or their employer(s)) 2021. Re-use permitted under CC BY-NC. No commercial re-use. See rights and permissions. Published by BMJ.

${ }^{1}$ AT Medics Ltd, London, UK ${ }^{2}$ School of Population Health and Environmental Sciences, King's College London, London, UK

Correspondence to Dr Veline L'Esperance; veline.lesperance@kcl.ac.uk

\section{ABSTRACT}

A quality improvement (QI) scheme was launched in 2017, covering a large group of 25 general practices working with a deprived registered population. The aim was to improve the measurable quality of care in a population where type2 diabetes (T2D) care had previously proved challenging. A complex set of $Q$ I interventions were co-designed by a team of primary care clinicians and educationalists and managers. These interventions included organisation-wide goal setting, using a datadriven approach, ensuring staff engagement, implementing an educational programme for pharmacists, facilitating web-based QI learning at-scale and using methods which ensured sustainability. This programme was used to optimise the management of T2D through improving the eight care processes and three treatment targets which form part of the annual national diabetes audit for patients with T2D. With the implemented improvement interventions, there was significant improvement in all care processes and all treatment targets for patients with diabetes. Achievement of all the eight care processes improved by $46.0 \%(p<0.001)$ while achievement of all three treatment targets improved by $13.5 \%(p<0.001)$. The QI programme provides an example of a data-driven largescale multicomponent intervention delivered in primary care in ethnically diverse and socially deprived areas.

\section{PROBLEM}

In the UK diabetes affects $9 \%$ of the adult population, with approximately $90 \%$ of cases classified as type 2 diabetes (T2D). ${ }^{1}$ Diabetes accounts for $10 \%$ of the total National Health Service (NHS) budget costing the NHS £10 billion a year, with $80 \%$ of this attributable to the treatment of complications. $^{2}$ Poorly managed T2D is associated with higher levels of complications and morbidity, including heart disease, stroke, diabetic retinopathy, kidney disease and amputation resulting in significant disability and premature mortality. ${ }^{34}$ Large inequality gaps relating to social deprivation have been demonstrated in diabetes outcomes, with increased preventable hospitalisations for diabetes complications noted in more deprived communities. ${ }^{5}$ Furthermore, the prevalence of T2D among ethnic minority groups is three to five times higher than the white European population. ${ }^{6}$ The estimated prevalence of diabetes in London $(9.1 \%)$ is higher than the national average $(8.7 \%),{ }^{7}$ however data from the National Diabetes Audit in 2016/2017 demonstrated that only $41 \%$ of patients with T2D in London achieved all treatment targets recommended by the National Institute for Health and Clinical Excellence (NICE). ${ }^{8}$ Reducing inequality in diabetes care is a major concern in line with the NHS Long-Term Plan recommendation to target healthcare interventions according to population need and to focus on prevention. ${ }^{9}$

The aim of this quality improvement (QI) programme was to improve measurable diabetes-clinical outcomes (eight care processes and three treatment targets) as defined by the National Diabetes Audit ${ }^{8}$ in a population where T2D care had previously proved challenging. We aimed to achieve a target of $75 \%$ for the eight care processes and $50 \%$ for the three treatment targets within 12 months. Each of these are described further in the Measurement Section.

\section{BACKGROUND}

General practice in the UK is seen as the cornerstone of the NHS with more than $90 \%$ of patient contacts being undertaken in general practice. A strong primary care-led system provides an ideal setting for the management of chronic diseases. However, there are many challenges to the primary care provision of high quality T2D care. These challenges include ineffective recalling 
of patients for annual diabetes review, variation in knowledge of clinicians of the components of the annual diabetes review and inconsistent adherence to best practice management guidelines. ${ }^{10-12}$ A systematic review of randomised trials of QI programmes demonstrated that they can result in measurable improvement in the quality of diabetes care and patient outcomes, particularly those that intervened on the entire system of chronic disease management were associated with the largest effects. ${ }^{13}$ The authors noted that QI strategies that aim to optimise the systems of care should (whenever feasible) be included in programmes to improve diabetes management, irrespective of glycated haemoglobin (HbA1c). ${ }^{13}$ In the UK, large scale QI programmes tend to focus on delivery within secondary care, while evidence for delivery within large scale primary care organisations is limited. However, in the $\mathrm{USA}^{14}$ and recently in Australia, ${ }^{15}$ large scale primary care QI programmes are evident through Patient-Centred Medical Homes. Given the UK government's agenda towards working at scale in primary care, ${ }^{16}$ methods for better delivery of clinical achievement at practice-level must be translatable across multiple sites. A previous study of 5910 patients with diabetes across 13 general practitioner (GP) practices demonstrated improvements in the percentage of patients achieving HbAlc (5\%), blood pressure (BP) $(6 \%)$ and total cholesterol $(4 \%) .{ }^{17}$ However, these improvements in T2D outcome were not sustained following completion of the project. ${ }^{17}$ Diabetes QI programmes also tend to focus on physicians, ${ }^{18}$ nurses $^{19}$ or pharmacists. ${ }^{17}$

Given the challenge of delivering high quality care for patients with T2D, a QI scheme was launched in 2017, covering a large group of general practices working with a deprived registered population.

\section{MEASUREMENT}

\section{Setting}

The practice organisation consisted of 25 GP practices across London caring for a population of over 240000 patients with $52 \%$ of those registered being from black and minority ethnic groups. ${ }^{20}$ Of the 9265 patients with a diagnosis of T2D $71 \%$ were from black and minority ethnic groups. Practices are distributed across socioeconomically and ethnically mixed populations, with some highly deprived communities. Staffing models for the practices include general practitioners, practice nurses, healthcare assistants, clinical pharmacists, a practice manager and administrative staff.

\section{Clinical outcomes measures}

We focussed on eight care process and three treatment targets which form part of the annual national diabetes audit for patients with T2D. ${ }^{8}$ These are outlined below:

\section{Care processes}

1. HbAlc measurement - blood test for measurement of glucose control
2. Serum creatinine measurement - blood test for measurement of kidney function

3. Cholesterol measurement - blood test for assessing cardiovascular risk

4. BP measurement - assessment of cardiovascular risk

5. Body mass index measurement - measurement of height and weight for assessment of cardiovascular risk

6. Foot examination - neurovascular assessment of foot.

7. Urine albumin:creatinine ratio measurement - urine test for assessment of early kidney disease

8. Record of smoking status - confirmation of smoking status for assessing cardiovascular risk.

\section{Treatment targets}

1. HbAlc of less than $59 \mathrm{mmol} / \mathrm{mol}$

2. BP of equal to or less than $140 / 80 \mathrm{~mm} \mathrm{Hg}$.

3. Total cholesterol of less than $5 \mathrm{mmol} / \mathrm{L}$

Data for each of these measurements across all practices were extracted using a population health management tool, EZ Analytics. This tool was specifically developed for this QI programme, further details of this tool is outlined in subsequent sections. Data extraction started in March 2017, these were then updated monthly thereafter. Each year GP practices are legally required to supply this data for their practice to NHS Digital for the National Diabetes Audit.

\section{DESIGN}

A complex set of interventions were co-designed by a team of primary care clinicians and educationalists and managers. The combination of interventions were based on best practice, incorporating published evidence and professional consensus. ${ }^{21}$ These interventions were aimed at improving diabetes care through targeting variability in clinical achievement across practices; ensuring consistent knowledge, education and training; and maintaining staff engagement in diabetes care. A key driver diagram, outlining primary drivers and strategies for improved diabetes care is displayed in online supplemental file 1.

The QI programme had multiple components which were targeted at health informatics, the workforce, education and training, processes and sustainability. Plan, Do, Study, Act (PDSA) cycles shown in figure 1 were used to trial the components of the improvement intervention implemented to achieve the aims. Each component outlined below was delivered across 25 practices.

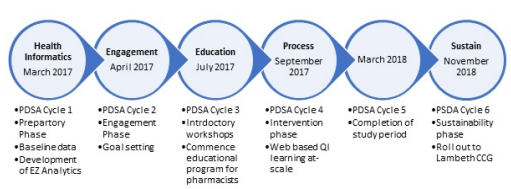

Figure 1 Timeline of the project illustrating when baseline data were collected, implementation of Plan, Do, Study, Act (PDSA) cycles and different phases of the project; preparatory, intervention and sustain. CCG, Clinical Commissioning Group; QI, quality improvement. 


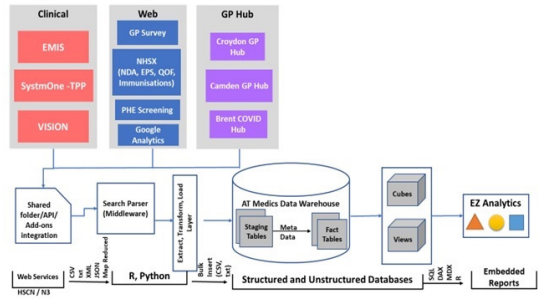

Figure 2 Data flow diagram. EPS, electronic prescription service; GP, general practitioner; EPS, national diabetes audit; PHE, public health england.

Health Informatics: A population health management tool, EZ Analytics was developed in March 2017 which integrates complex data from any data source, into user friendly information. This platform was designed to identify areas which required optimisation in chronic disease areas. EZ Analytics enabled staff to view performance of patient outcomes at individual practice, regional and national levels. This tool was used to capture baseline data and ongoing progress throughout the QI programme. The use of data for benchmarking against recommended targets and performance of other clinicians has been noted as a motivating factor for driving QI strategies. ${ }^{22}$ Using data-driven approaches outside the research setting is often restricted by the practical constraints of achieving near real-time capture of structured clinical data. Furthermore, patient-level data available through electronic health records creates challenges given the volume, variety and speed at which the data is generated. The tool comprises over 600 clinical data sets. The stand-alone Diabetes Application was able to interrogate National Diabetes Audit (NDA) data alongside the real-time extraction of in-house performance data. Data process flow is demonstrated in figure 2. Data-driven approaches to QI have been demonstrated in areas such as cardiovascular care ${ }^{23}$ but have been limited in informing QI programmes in primary care.

Staff Engagement: Goal setting was initiated in April 2017 to ensure that staff members were aware of the need for improvement and to gather consensus on developing this. A series of initial workshops were also undertaken to develop ideas and explore ways of overcoming potential challenges. Pharmacists, administrative assistants and practice managers formed core members of the implementation team. Multidisciplinary staff engagement formed an integral component to this QI programme. Multidisciplinary engagement across the organisation also formed a key basis for the strategic direction of this QI programme. ${ }^{24} 25$

\section{Educational programme for pharmacists}

Underpinning the QI initiative was a comprehensive educational programme for practice pharmacists. This bespoke programme for 25 clinical pharmacists was delivered at-scale by AT Learning. AT Learning is a modularised approach to training of pharmacists in general practice in line with the ambitions of the GP Five Year
Forward View. ${ }^{16}$ The educational programme consisted of web-based teaching and clinical scenarios.

\section{Process understanding}

Standardised processes for diabetes checks were developed and disseminated to all practices. This multidisciplinary team-based QI training programme ensured all team members understood the processes and current guidance for diabetes care. This guidance was distributed via webbased learning sessions for administrative assistants, practice managers and clinicians, explaining processes and targets.

\section{STRATEGY}

Our SMART aim was to improve measurable diabetes patient outcomes as defined by the National Diabetes Audit including the eight care processes and three clinical targets. We undertook four PDSA test cycles in order to achieve this.

\section{PDSA cycle 1: health informatics}

EZ Analytics was developed to ensure that this QI Programme maintained a data-driven approach. To identify variation in service provision and patient outcomes at-scale, a data analytics tool, EZ Analytics ${ }^{26}$ was developed. EZ Analytics incorporates key performance data from all clinical systems including EMIS, SystmOne and Vision. EZ Analytics identifies areas of variation and parameters for benchmarking practices against local and national outcomes. In March 2017, all practices were given access to EZ Analytics via an online log-in. This enabled practices to view their performance compared with other practices locally, regionally and nationally. Updates of each practice's performance were available monthly. The key strategies for ensuring staff used this tool were data democratisation and data socialisation.

Data democratisation-the process of making data accessible, formed a key component of this QI strategy. EZ Analytics is available online to all team members across the organisation which allows the data to be data easily accessible, empowers team members to be data custodians and data analysts and to take collective responsibility for the data.

Data socialisation was integral to ensuring team members remained engaged and actively contributed to this QI programme. To ensure that data-driven insights were available to everyone in a self-service manner, each employee could readily access the data and data analytics tools enabling them to benchmark their own practices against local, region and national comparators.

\section{PDSA cycle 2: engagement}

Following development of the EZ Analytics, the team was able to use the data to establish the baseline clinical achievement for the NDA patient outcomes for each practice. This baseline data was used for organisationwide goal setting. The main goals consisted of improving measurable clinical outcomes in diabetes care, achieving 
a target of $75 \%$ for the eight care processes and $50 \%$ for the three treatment targets. Together, the lead GPs, pharmacists and practice managers jointly established and prioritised the challenges facing diabetes management across the organisation. This resulted in selection of QI interventions supported by a broad range of clinical and non-clinical healthcare professionals. Collaboration was integral to the development and implementation of this intervention. The objectives for this QI programme were clearly defined, prioritised by the organisation and distributed across all practices and conveyed to staff. These were then formally launched through introductory webinars, followed by goal setting events and workshops, then monthly team meetings.

Building on the engagement activities a series of initial workshops were also undertaken to develop ideas and explore ways of overcoming potential challenges. Pharmacists, administrative assistants and practice managers formed core members of the implementation team.

\section{PDSA cycle 3: educational programme for pharmacists}

Following the implementation of engagement strategies, it was noted that more training was required, particularly for the pharmacists who were undertaking the patient assessments. These web-based clinical tutorials were based on the NICE Diabetes guidelines. ${ }^{27}$ Additional practical supervision during diabetic checks and random case analysis with feedback were provided by the GP lead (TR) and pharmacist lead (YA). Performance was reviewed through annual Objective Structured Clinical Examination and multiple-choice questions which were devised by the lead GP (TR), an approved GP trainer. Continuing professional development certificates were provided for each activity.

\section{PDSA cycle 4: process}

PDSA cycle 4 focussed on ensuring that processes were place and working smoothly to achieve the eight diabetes care processes and three treatment targets. This was undertaken through the use of web-based QI learning at-scale. Sessions had up to 80 attendees at a time and for those unable to attend, web-based communication was recorded for viewing at a later time. There were three sessions over the 12-month period, each lasting 2 hours.

Monthly web-based catch up meetings were also implemented across all practices for pharmacists, administrative assistants and practice managers. These monthly webinars enabled everyone to understand how their role contributed to the QI programme, reflect on the clinical achievement of their practice compared with other practices and share learning from practices who were on target with their achievement.

During these sessions, each practice was appraised based on their latest performance, based on near real-time data provided from EZ Analytics (figure 3). This ensured that performance data played a key role in improving the diabetes outcomes. Each month the leading practice received recognition for their success.

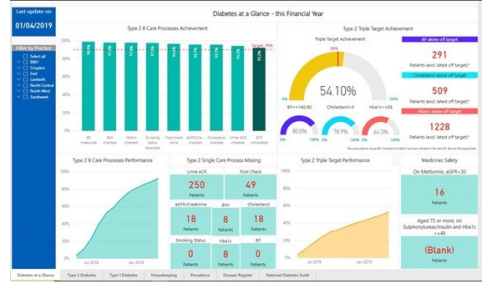

Figure 3 Output from EZ Analytics.

\section{PDSA cycle 5: review and improvement of interventions}

In this cycle we focussed on reviewing all of the interventions within the previous cycles and made improvements where necessary. This period mainly encompassed motivating and encouraging staff through developing competition between practices to improve their targets. Practice managers facilitated regular recalls and reviews to be undertaken by the multidisciplinary staff consisting of healthcare assistants, nurses and pharmacists. The same materials were used across all practices ensuring a standardised approach was applied to all practices.

\section{PDSA cycle 6: sustainability and generalisability to other practices}

Following achievement of sustainability within AT Medics practices, this QI programme was extended across all 40 practices within Lambeth Clinical Commissioning Group in November 2018. Lambeth Clinical Commissioning Group has a total of 394055 registered patients, of which 17115 have a diagnosis of T2D. The main objective of this initiative was an overall improvement in the eight care processes and three treatment targets. The intervention included use of EZ Analytics as well as webinars targeted at administrative assistants, practice managers and pharmacists. Three newsletters were also distributed to each practice during the implementation period. After 4 months (March 2019), preliminary data for these practices were collected.

\section{Statistical analysis}

Analysis was performed on all outcomes using Stata V.16. Four time periods were constructed for each outcome variable: (1) 15 months - March 2016 and (2) 3 months prior to the QI intervention - March 2017 (baseline); (3) 9 months - March 2018 (end of intervention of intervention (period); and (4) 21 months after the QI intervention was implemented. Outcomes before and after were compared using two-tailed t-test. P value $<0.05$ was considered statistically significant. Given the small amount of missing data $(4.8 \%)$, we conducted a complete case analysis.

\section{Patient involvement}

In July 2019 patients with T2D were invited via text to provide their feedback regarding their experiences of T2D management by the practice. The patients completed a short online questionnaire regarding the care they received for T2D. In total 543 patients with T2D completed the short questionnaire. 


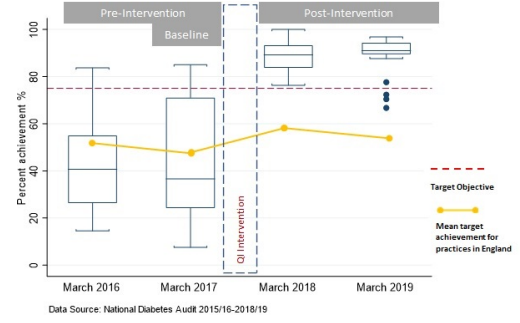

Figure 4 Achievement of type 2 diabetes - eight care processes 2015/2016 to 2018/2019. QI,quality improvement.

\section{RESULTS}

Results of the QI intervention are shown in figures 4 and 5 in line with the annualised National Diabetes Audit results. Additionally, monthly data monitoring results are shown in online supplemental figure 1) and online supplemental figure 2). The online supplemental table 1) also shows the breakdown of improvement across each individual outcome measure. The box plots in figures 4 and 5 demonstrate that at baseline $(2016 / 2017)$ there was wide variation between the highest and lowest performing practices achieving their eight care processes (figure 4) and three treatment targets (figure 5). In the baseline period the lowest achieving practice attained $8 \%$ achievement for their eight care processes and $29 \%$ for the three treatment targets, compared with the highest performing practice of $85 \%$ and $52 \%$, respectively. Following the intervention, there was a significant improvement in achievement across all care processes and treatment targets. Reduction in variation between practices was also noted as a key improvement. The dashed red line denotes the agreed objective of $75 \%$ achievement for the eight care process and $50 \%$ achievement for the three treatment targets. Both these goals were met by 22 out of the 25 practices by $2018 / 2019$.

With the implemented improvement interventions, there was significant improvement in all care process and all treatment targets for patients with diabetes. Achievement of all the eight care processes improved by $46.0 \%$ $(p<0.001)$ while achievement of all three treatment targets improved by $13.5 \%(\mathrm{p}<0.001)$. The improvements in achievement demonstrated following this QI intervention were not replicated nationally across practices in England over the study period. The yellow lines in figures 4 and 5 demonstrate the mean achievement for practices in England. The mean achievement for practices in England for the eight care process from March 2017 to

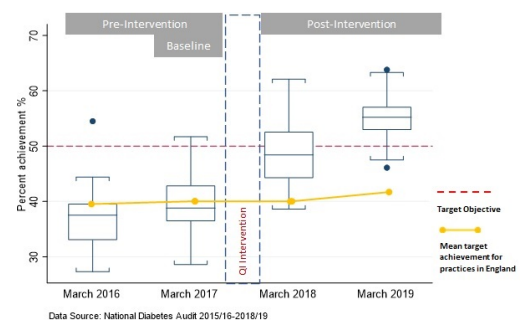

Figure 5 Achievement of type 2 diabetes - three treatment targets 2015/2016 to 2018/2019. QI,quality improvement.
March 2018 increased by only $11.1 \%$ ( $47.7 \%$ to $58.8 \%$ ), however over the same period achievement of three treatment targets reduced by $1 \%$ ( $41.1 \%$ to $40.1 \%)$. In March 2019, achievement in the eight care processes for practices in England reduced by $4.5 \%$ while achievement in the three treatment targets reduced increased by only $1.6 \%(40.1 \%$ to $41.7 \%)$. The figures demonstrate that following the QI intervention practices within the organisation achieved greater improvement across all three treatment targets and eight care processes compared with the mean achievement seen in practices in England.

Online supplemental table 1 provides the per cent achievement for each care process and treatment target across 4 years. The greatest improvement noted in the eight care processes was in the measurement of urine albumin:creatinine ratio measurement $(35.1 \%, \mathrm{p}<0.001)$. Of the three treatment targets, BP control of less than or equal to $140 / 80 \mathrm{~mm} \mathrm{Hg}$ had the largest improvement in achievement $(10.8 \%, \mathrm{p}<0.001)$. These improvements were also clinically significant and were also sustained in the year following the study period.

Results of the patient survey noted that $81 \%$ of patients felt involved in their diabetes care and $80 \%$ felt supported by their practice in terms of their diabetic care. Although, $39 \%$ agreed that the level of diabetic care from their practice improved in the previous year, $43 \%$ felt their care remained the same. Seventy-six per cent of patients felt that they were invited for diabetic review in a timely manner, while $71 \%$ agreed they were provided with relevant information to support their diabetes care. Overall, $71 \%$ would recommend this service to other patients with diabetes.

\section{Application to other practices}

Following roll-out of this QI programme to Lambeth Clinical Commissioning Group in November 2018, practices demonstrated an improvement in achievement of the eight care processes $(62.5 \%$ to $77.1 \%)$ and the three treatment targets $(40.0 \%$ to $44.6 \%)$.

\section{LESSONS AND LIMITATIONS}

This QI programme demonstrated significant improvement in T2D outcomes in primary care for the eight care processes and three treatment targets. These improvements were also greater compared with practices nationally. This programme also demonstrated greater achievement in BP (11\%) and total cholesterol (6\%) compared with a previous London based study ( $6 \%$ and $4 \%$, respectively). ${ }^{17}$ Although both studies achieved similar improvements in HbAlc targets of approximately $5 \%$, improvements in this QI programme was sustained following the end of the study period. Another QI study ${ }^{28}$ in London demonstrated an improvement of $40.3 \%$ in the uptake of eight care processes, however this was less than the improvement seen among our practices of $46 \%$. Our QI intervention also demonstrated a greater improvement in the three treatment targets (13.5\% vs 11.7$)$. Additionally, 
our QI intervention sought no additional funding and did not provide any financial incentive to practices.

The delivery of QI programmes requires review of existing research, effective leadership, engagement with staff. However, this QI programme also incorporated relevant education and training for clinical pharmacists and used a data-driven approach. Diversification of workforce was a key driver in the success of this programme which has not been demonstrated previously in large scale primary care QI programmes. QI programmes tend to rely heavily on clinicians. However, in this programme the role of administrative team who are often overlooked in primary care team was essential. Furthermore, unlike other diabetes QI programmes, GPs involvement was limited, enabling them to focus on other aspects of primary care delivery. Instead, pharmacists were integrated into the team, reviewing the data and clinically assessing patients. This is particularly important with the UK context, given the current UK drive to use clinical pharmacists more effectively in primary care. ${ }^{29}$

Using a standardised approach, the outcomes following the QI intervention have demonstrated they are generalisable and scalable when applied to other practices within London. After this programme was shown to be sustainable following the end of the study period in AT Medics practices, it was rolled out to practices within Lambeth (PDSA cycle 6) which demonstrated improvement in the care processes and treatment targets. The challenges faced in delivery good diabetes care included ineffective recalling, variable clinical knowledge regarding components of annual reviews, variation in completeness of care during annual reviews and inconsistent clinical management of BP, cholesterol and HbA1c. Using health informatics tools we were able to interrogate our practice data easily to better understand the variation between practices. The use of health informatics and electronic health records has been cited in the literature as a facilitator in the delivery of higher quality diabetes care in primary care. ${ }^{30}$ This use of data analytics and knowledge was shared widely across the organisation and ensured that all team members had easily accessible to data and fostered a culture of data custodians among the staff. The foundation of this QI programme is based on effective communication through accessible means such as webinars and newsletters, and continuous efficient dissemination of healthcare data across all team members. The availability of these tools is integral in enabling this QI programme to be applied to other patient groups and healthcare systems.

People with diabetes in deprived communities often do not attain the health assessments needed to prevent diabetes complications. They are less likely to have their blood pressure, blood glucose levels or weight checked. This QI programme aimed to ensure that strategies were put in place to recognise underachievement in practices, provide training and educational development of staff and maintain engagement of the team in a multidisciplinary approach.
Given the resource challenges faced in primary care, this QI programme relied on the use of clinical pharmacists, a population health management tool and administrative staff. Therefore, this relieved the administrative burden from GPs, enabling them to focus on other clinical duties. Having the pharmacists work centrally allowed their expertise to be distributed across areas of greater clinical need. As there was no additional funding for the QI programme, developing a standardised approach was key to ensuring that QI programme could be applied to different settings. Many of the principles applied to this QI programme such as leadership, use of health informatics, education and training and staff engagement are transferable to other settings. However, we recognise there are also challenges in developing QI programmes such as time and the availability of in-house personnel with the requisite informatics and educational skills.

Observational studies can only provide associations without proven causality and require further study with a rigorous evaluation strategy. It is possible that factors external to our intervention contributed to the observed results; however, the lack of similar reductions nationally suggests that our intervention may be responsible for the observed improvements across our practices.

\section{CONCLUSION}

The QI programme provides an example of a data-driven large-scale multicomponent intervention delivered in primary care in ethnically diverse and socially deprived areas. Clinical pharmacists, administrative assistants and practice managers played a role in the implementation and delivery. Overall, this QI programme enabled a sense of ownership by the whole practice team. This programme has demonstrated sustainability in the improvement of the care of patients with diabetes. Further study and evaluation are warranted.

Acknowledgements We would like to thank the clinical and administrative teams across all AT Medics practices who made this project possible.

Contributors The Quality Improvement Project was designed and delivered by TR, YA, OD, MT and TK. The data guarantor is AE. The first draft was written by TR and VL. Data extraction was undertaken by AE and TK. Data analysis was undertaken by VL and reviewed by PS. All authors reviewed and contributed to the final draft.

Funding The authors have not declared a specific grant for this research from any funding agency in the public, commercial or not-for-profit sectors.

Competing interests None declared.

Patient consent for publication Not required.

Ethics approval Ethical approval was not required as the work was considered to be a service-based improvement programme and no identifiable confidential information was collected. All information was managed according to UK NHS information governance requirements. All clinical data had all patient identifiers removed and was fully anonymised.

Provenance and peer review Not commissioned; externally peer reviewed.

Data availability statement Data are available in a public, open access repository.

Supplemental material This content has been supplied by the author(s). It has not been vetted by BMJ Publishing Group Limited (BMJ) and may not have been peer-reviewed. Any opinions or recommendations discussed are solely those of the author(s) and are not endorsed by BMJ. BMJ disclaims all liability and responsibility arising from any reliance placed on the content. Where the content 
includes any translated material, BMJ does not warrant the accuracy and reliability of the translations (including but not limited to local regulations, clinical guidelines, terminology, drug names and drug dosages), and is not responsible for any error and/or omissions arising from translation and adaptation or otherwise.

Open access This is an open access article distributed in accordance with the Creative Commons Attribution Non Commercial (CC BY-NC 4.0) license, which permits others to distribute, remix, adapt, build upon this work non-commercially, and license their derivative works on different terms, provided the original work is properly cited, appropriate credit is given, any changes made indicated, and the use is non-commercial. See: http://creativecommons.org/licenses/by-nc/4.0/.

\section{REFERENCES}

1 NHS Digital. National diabetes audit -2017-2018: report 1, care processes and treatment targets, 2019. Available: https://digital.nhs. uk/data-and-information/publications/statistical/national-diabetesaudit/report-1-care-processes-and-treatment-targets-2017-18-shortreport

2 Dlabetes UK. The cost of diabetes report, 2014.

3 , Sarwar N, Gao P, et al, Emerging Risk Factors Collaboration. Diabetes mellitus, fasting blood glucose concentration, and risk of vascular disease: a collaborative meta-analysis of 102 prospective studies. Lancet 2010;375:2215-22.

4 Danaei G, Finucane MM, Lu Y, et al. National, regional, and global trends in fasting plasma glucose and diabetes prevalence since 1980: systematic analysis of health examination surveys and epidemiological studies with 370 country-years and $2 \cdot 7$ million participants. Lancet 2011;378:31-40.

5 Fleetcroft R, Asaria M, Ali S, et al. Outcomes and inequalities in diabetes from 2004/2005 to 2011/2012: English longitudinal study. Br $J$ Gen Pract 2017;67:e1-9.

6 Goff LM. Ethnicity and type 2 diabetes in the UK. Diabet Med 2019;36:927-38.

7 Public Health England. National cardiovascular intelligence network: prevalence estimates of diabetes by clinical commisioning group (CCG) and England. public health England, 2015. Available: https:// www.gov.uk/government/publications/diabetes-prevalenceestimates-for-local-populations

8 NHS Digital. National diabetes audit -2016-2017: report 1, care processes and treatment targets, 2017. Available: https://digital. nhs.uk/data-and-information/publications/statistical/nationaldiabetes-audit/national-diabetes-audit-report-1-care-processes-andtreatment-targets-2016-17

9 NHS England. The NHS long term plan. NHS England, 2019. Available: https://www.longtermplan.nhs.uk/publication/nhs-longterm-plan/

10 Ye J, Zhang R, Bannon JE, et al. Identifying practice facilitation delays and barriers in primary care quality improvement. J Am Board Fam Med 2020;33:655-64.

11 Haw JS, Narayan KMV, Ali MK. Quality improvement in diabetes-successful in achieving better care with hopes for prevention. Ann N $Y$ Acad Sci 2015;1353:138-51.
12 Rushforth B, McCrorie C, Glidewell L, et al. Barriers to effective management of type 2 diabetes in primary care: qualitative systematic review. Br J Gen Pract 2016;66:e114-27.

13 Tricco AC, Ivers NM, Grimshaw JM, et al. Effectiveness of quality improvement strategies on the management of diabetes: a systematic review and meta-analysis. Lancet 2012;379:2252-61.

14 Jabbarpour Y, DeMarchis E, Bazemore A, et al. The impact of primary care practice transformation on cost, quality, and utilization: a systematic review of research published in 2016, 2017. Available: https://www.pcpcc.org/resource/impact-primary-care-practicetransformation-cost-quality-and-utilization

15 Metusela C, Usherwood T, Lawson K, et al. Patient centred medical home $(\mathrm{PCMH})$ transitions in Western Sydney, Australia: a qualitative study. BMC Health Serv Res 2020;20:285.

16 NHS England. General practice five year forward view. NHS England: Leeds, 2016. https://www.england.nhs.uk/wp-content/uploads/2016/ 04/gpfv.pdf

17 Langran T, Nanda N, Bataveljic A, et al. Supporting the management of type 2 diabetes with pharmacist-led reviews: an observational analysis. BMJ Open 2017;7:e013451.

18 Foy R, Hempel S, Rubenstein L, et al. Meta-Analysis: effect of interactive communication between collaborating primary care physicians and specialists. Ann Intern Med 2010;152:247-58.

19 Clark CE, Smith LFP, Taylor RS, et al. Nurse-Led interventions used to improve control of high blood pressure in people with diabetes: a systematic review and meta-analysis. Diabet Med 2011;28:250-61.

20 AT Medics. Where we work, 2020. Available: https://www.atmedics. com/where-we-work/

21 van Bokhoven MA, Kok G, van der Weijden T. Designing a quality improvement intervention: a systematic approach. Qual Saf Health Care 2003;12:215-20.

22 Braga MFB, Casanova A, Teoh $\mathrm{H}$, et al. Poor achievement of guidelines-recommended targets in type 2 diabetes: findings from a contemporary prospective cohort study. Int J Clin Pract 2012;66:457-64.

23 Rumsfeld JS, Joynt KE, Maddox TM. Big data analytics to improve cardiovascular care: promise and challenges. Nat Rev Cardiol 2016;13:350-9.

24 Taitz JM, Lee TH, Sequist TD. A framework for engaging physicians in quality and safety. BMJ Qual Saf 2012;21:722-8.

25 Pannick S, Sevdalis N, Athanasiou T. Beyond clinical engagement: a pragmatic model for quality improvement interventions, aligning clinical and managerial priorities. BMJ Qual Saf 2016;25:716-25.

26 AT Tech. Ez analytics, 2020. Available: https://ezanalytics.co.uk/

27 NICE. Type 2 diabetes in adults: management: NICE guideline [NG28], 2019.

28 Gupta A, Baldwin A, Sood J, et al. Diabetes quality improvement at scale: how Barking and Dagenham, Redbridge, and Havering CCGs tackled the 'Seven Spreadly Sins'. Br J Gen Pract 2020;70:40-1.

29 Royal College of General Practitioners and Royal College of General Practitioners. Joint statement: pharmacists set to work in GP surgeries in radical move to ease pressures on general practice and improve patient care, 2015.

30 Riordan F, McHugh SM, O'Donovan C, et al. The role of physician and practice characteristics in the quality of diabetes management in primary care: systematic review and meta-analysis. J Gen Intern Med 2020;35:1836-48. 